\title{
Frações, sua representação decimal e a calculadora
}

\author{
Dora Kindel Erika Favoretto
}

\begin{abstract}
Resumo
O uso de calculadoras nas aulas de matemática, principalmente no ensino fundamental, ainda tem sido pouco explorado. Neste artigo, pretende-se discutir uma atividade proposta em turmas do $8^{\circ}$ ano de diferentes escolas do Rio de Janeiro. Objetiva-se refletir sobre as representações decimais das frações com denominadores 2, 4, 8 e 16 e desenvolver o cálculo mental por meio da observação de regularidades e da comparação da representação decimal com os denominadores ou com os numeradores das frações em questão. Os alunos utilizaram calculadoras, pelo menos uma por dupla, e trabalharam agrupados em trios ou quartetos. Os resultados registrados individualmente nos cadernos deveriam apresentar as justificativas discutidas no grupo. No final da aula, dois tempos de 50 minutos, as respostas dos alunos foram escritas no quadro-negro e, novamente, discutidas e sistematizadas. A análise das respostas revelou que os alunos apresentavam explicações detalhadas sobre os procedimentos, com justificativas às suas observações.
\end{abstract}

Palavras-chave: calculadora; números decimais; frações ordinárias, investigação em sala de aula.

\begin{abstract}
The use of calculators in mathematics classes, mainly in elementary school, has still been little explored. In this article, we intend to discuss an activity proposed in 8th grade classes from different schools in Rio de Janeiro. The objective of this paper is to reflect on the decimal representations of fractions with denominators 2, 4, 8 and 16 and to develop the mental calculation by observing regularities and comparing the decimal representation with the denominators or numerators of the fractions in question. Students used calculators, at least one per couple, and worked grouped by trios or quartets. The results recorded individually in the notebooks should present the justifications discussed in the group. At the end of the class, two 50-minute periods, the students' responses were written on the chalkboard and again discussed and systematized. The analysis of the answers revealed that the students presented detailed explanations about the procedures, with justifications for their observations.
\end{abstract}

Keywords: calculator; decimal numbers; ordinary fractions, classroom research.

\section{Introdução}

O desenvolvimento social do homem, através dos tempos e em diferentes contextos, faz evoluir tanto o conceito dos números quanto os cálculos necessários para com eles operar. Isso se justifica no que toca à necessidade da resolução dos problemas do cotidiano e no que se refere à evolução científica e tecnológica.

\footnotetext{
${ }^{1}$ Este artigo é fruto da análise de material coletado durante o período do mestrado realizado em 1998.
} 
Para interpretar e atuar no mundo, desenvolvem-se cálculos extraordinariamente complexos, os quais nem sempre são memorizados por nós. Assim, a fim de facilitar tais cálculos morosos e complicados, o homem cria os mais variados instrumentos de cálculo, como é o caso das calculadoras. Apesar dos diferentes modelos de calculadoras existentes no mercado hoje, seu uso no ensino tem ficado restrito às disciplinas de natureza técnica ou aos cursos técnicos e científicos.

Nas aulas de matemática, sobretudo no ensino fundamental, com objetivo metodológico, a calculadora tem sido muito pouco usada, apesar de se tratar de um instrumento que proporciona a exploração de novas estratégias e novos métodos de trabalho. Um dos argumentos usados é o de que o uso da calculadora impede os alunos de saber a tabuada, o que não se confirmou: ,

\section{(...) ao contrário do que se imagina, os alunos passaram a saber a tabuada de cor, mesmo não sendo este o objetivo da atividade, e aqueles que apresentavam dificuldade melhoraram seu desempenho.(...). Alunos que não sabiam a tabuada buscaram encontrar estratégias de cálculo mental que servisse como forma de agilizar o cálculo com a calculadora. [3, p60]}

Embora as pesquisas que envolvem as frações sejam assunto recorrente, existem poucos trabalhos usando a calculadora para esse fim. Assim, pretendem-se apresentar, neste artigo, os resultados obtidos a partir de uma experiência com o uso de calculadoras em turmas da antiga $7^{\text {a }}$ série, atual $8^{\mathrm{o}}$ ano, de uma escola da zona sul do Rio de Janeiro. No trabalho, foi proposta uma série de atividades para discutir os raci-onais visando: a) verificar de que maneira os alunos estabelecem comparações entre a representação ordinária de frações e suas correspondentes representações decimais; b) identificar de que forma os alunos identificam e agrupam as regularidades observadas; c) verificar que procedimentos e estratégias os alunos usam para generalizá-las. Para tanto, as atividades com questões de caráter aberto, isto é, questões do tipo "o que acontece se..." foram apresentadas. Esse tipo de trabalho sustenta-se na proposta da investigação em sala de aula apresentada por Ponte (2003). [6]

O trabalho foi desenvolvido em dois tempos de 50 minutos cada. Para o desenvolvimento da proposta, os alunos foram organizados em grupos de três ou quatro e foi entregue pelo menos uma calculadora para cada dupla. Todos os alunos deveriam anotar as frações e suas representações decimais no caderno e discutir as justificativas até um consenso entre os integrantes do grupo, para, ao final, um relator apresentar as conclusões oralmente para a turma. Vale ressaltar, ainda, que os estudantes estavam acostumados a desenvolver trabalhos em grupos e realizar pesquisas para observar regularidades numéricas.

A vivência dos alunos, em diferentes momentos de aprendizagem_a pesquisa individual, a discussão sobre os pontos de vista, a troca de informações, a apresentação e a defesa das descobertas feitas pelos grupos durante a apresentação oral_favoreceu o diálogo e a busca de um consenso no interior dos grupos para as respostas.

A análise dos relatórios individuais entregues à professora revelou detalhes interessantes sobre a forma como cada aluno busca sintetizar, explicar e detalhar as descobertas e o procedimento seguido durante o trabalho investigativo. Neste trabalho, pôde-se evidenciar ainda a riqueza de detalhes possíveis a ser descoberta pelos alunos quando inseridos em um ambiente de estudo em que se promove o diálogo, a discussão e a reflexão sobre os diferentes pontos de vista em busca de um consenso para resolver uma situação problema, o que, no nosso caso, é um contexto de matemática pura.

\section{As frações e os números decimais}

Segundo Breke, [1], os alunos, quando em processo de aprendizagem, entendem os números decimais como um par de números inteiros: um que vem antes da vírgula e o outro escrito após a vírgula.

Nos livros didáticos, a fração é, usualmente, definida como parte do todo. No caso, o numerador representa as partes que foram tomadas do inteiro, e o denominador representa em quantas partes o inteiro foi dividido. 
Para encontrar a representação decimal de uma fração, dividem-se suas partes componentes - numerador e denominador -, ou seja, a fração é vista como divisão entre dois números inteiros.

Desse modo e em síntese, como hipótese de trabalho, parte-se da ideia de fração como divisão entre dois números inteiros, para propor uma intervenção em uma sala de aula do oitavo ano, já que, nas calculadoras que usamos, não é possível representar uma fração em sua forma ordinária: $a / b \operatorname{com} b \neq 0$. Com a atividade proposta, visa-se refletir sobre a conexão entre a representação de um número racional na sua forma ordinária e a sua representação decimal.

Neste trabalho, será apresentada a análise de um recorte das atividades de frações com denominado-res pertencentes às sequências $\{2,4,8 \ldots\}$ e $\{5,25,125 \ldots\}$ e as relações entre elas. O objetivo da atividade era apresentar situações problemas de tal forma que os estudantes obtivessem uma gama razoável de elementos para análise, comparando os resultados, e que, os mesmos, desenvolvessem estratégias de generalizações. Para desenvolver o trabalho, os alunos, em pequenos grupos (no máximo quatro componentes), usavam pelo menos uma calculadora por dupla. Foram propostas atividades investigativas, entendidas aqui como aquelas que apresentam questões abertas do tipo "O que acontece se...?". No caso, apresentaram-se as seguintes questões:

a) $\mathrm{O}$ que acontece se transformarmos as frações com denominador dois em números decimais?

b) E nas frações com denominador quatro?

c) Verifique também o que acontece com as frações cujos denominadores são iguais à 8, 16 e assim por diante.

\section{Quadro 1: Atividade apresentada aos alunos}

As respostas e os procedimentos foram analisados conforme descrição apresentada a seguir.

\section{Procedimentos e análise}

\subsection{As metades}

A primeira atividade foi a de identificar o que acontece quando transformamos as frações $\{1 / 2,2 / 2,3 / 2$, $4 / 2,5 / 2 \ldots\}$ em números decimais. Em nosso caso, tratou-se de dividir qualquer número natural por dois. A dificuldade inicial discutida pelos alunos dizia respeito ao procedimento que deveria ser usado com a calculadora. Vale ressaltar que muitos alunos tinham conhecimento de que $1 / 2$ poderia ser operado pelo algoritmo da divisão e, mesmo assim, eles não estabeleceram conexão entre essa ideia e o fato de que agora bastava apertar as teclas:

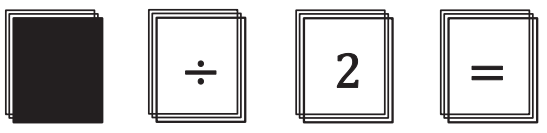


Diante dessa dificuldade e do fato de que a atividade assim apresentada não foi autoexplicativa - foi necessária a intervenção da professora em todos os grupos -, a questão foi modificada para:

a) O que acontece se dividirmos qualquer número natural $n$ por dois?

\section{Quadro 2: continuação da atividade}

Depois que os alunos compreenderam a atividade e identificaram o procedimento necessário para en-contrar a representação decimal de uma fração ordinária, com ou sem uso da calculadora, foram listadas várias frações com denominador dois. Logo, a partir da leitura dos resultados no visor da calculadora, os alunos identificaram as regularidades abaixo elencadas e assim justificadas por $\operatorname{eles}^{2}$ :

A - Se o numerador for um número par, então, o resultado será um número inteiro;

B - Se o numerador for ímpar, logo, o resultado não será um número inteiro;

C - Se o numerador for ímpar, logo, o resultado será um número quebrado;

D - Se o numerador for ímpar, logo, o resultado será "um ponto cinco".

Nessa investigação, alguns alunos também compreenderam o número decimal como um par de números (Resposta D). Esse tipo de resposta foi muito mais recorrente do que aquilo que esperávamos de uma turma de $8^{\circ}$ ano, pois pelo menos um dos integrantes de cada grupo a evidenciou e, de um modo geral, foi aceita pelos demais.

Essas respostas podem ser sistematizadas em duas categorias: uma que considera o número inteiro (em nosso caso, natural) como referência; e outra que considera o resultado como um número não inteiro, número decimal, e que foi entendido como "número quebrado".

Para dar continuidade à investigação, propôs-se que os alunos continuassem a efetuar as divisões usando como denominadores os números da sequência $\{4,8,16 \ldots\}$ e verificassem o que estava acontecendo com os "números quebrados", números decimais. Apresentam-se a seguir os resultados obtidos para a pes-quisa feita com os quartos, com os oitavos e com uma tentativa de generalização das regularidades observadas.

\subsection{Os quartos}

Para as frações com denominadores iguais a quatro, os alunos disseram que existiam muitas e verifi-caram que havia semelhanças entre a parte decimal de algumas frações, conforme podemos ver a seguir:

A- "Nos quartos?! Aí dá um monte! Assim: 'ponto vinte e cinco, ponto cinco, ponto setenta e cinco e inteiro.'"

B- "Quando o numerador é múltiplo de quatro, então dá inteiro; se não é múltiplo de quatro, então dá quebrado."

C- "Se o numerador for menor do que quatro dá zero vírgula um número."

D- "Ih, é! E se o numerador for maior do que quatro dá todos os números antes da vírgula e depois dá tudo igual."

E- "Nem sempre. Oh, dá um monte com ponto cinco, um monte com ponto vinte e cinco. Hummmm."

Os alunos D e E trocam pontos de vista distintos e se veem em um impasse para relatar a descoberta.

\footnotetext{
${ }^{2}$ Para preservarmos a identidade dos alunos optamos por elencar as respostas por letras do alfabeto.
} 
Nas respostas dadas pelos alunos sobre os oitavos e sobre os demais denominadores, foi recorrente o tipo de resposta dada anteriormente. Ou seja, agrupavam em respostas pela representação decimal. Para o caso em que o denominador era 16, observamos que os alunos não fizeram muitas operações associando a fração ordinária com o número decimal correspondente. Para esse caso, os alunos afirmaram que só precisam fazer do um ao quinze no numerador, pois os demais terão os mesmos algarismos na parte decimal.

No caso desse trabalho, ser múltiplo do denominador foi a referência usada. Assim, o conjunto das frações $n / 2^{b}$ foram divididos em dois grupos:

\begin{tabular}{|l|l|}
\hline Numerador múltiplo do denominador & Numerador não múltiplo do denominador \\
\hline
\end{tabular}

Quadro 3: Classificação das frações

$$
\begin{gathered}
U=\text { conjunto das frações } \frac{n}{2^{b}} \\
n-\mathrm{n}^{\circ} \text { natural }
\end{gathered}
$$

$b \in\{1,2,3,4 \ldots\}$ também um número natural.

$$
U=\left\{x \in \mathbb{Q}^{+} \mid x=\frac{n}{2^{b}}\right\}
$$

Como transcender a visão que define o conjunto de objetos dessa forma e que, para os alunos, é sufi-ciente? Eles partem do que é real para si, daquilo que é objeto de seu conhecimento, como no caso "é ser múltiplo de", e aplicam esse conhecimento em um novo contexto. Nesse caso, aplicam o conceito de múltiplo ao universo das frações. Diante disso, como fazê-los analisar o conjunto de frações cujo resultado não é inteiro?

Ora, se o conjunto está dividido em duas partes complementares de tal forma que é possível denominá-las tendo como referência a propriedade comum a seus elementos, podemos, então, ter os seguintes subconjuntos: o conjunto formado pelos elementos cujo resultado é um número inteiro e o conjunto formado pelos elementos formados por "números quebrados" (definição dada pelos alunos) ou por um decimal exato (definição dada pelo professor) e trabalhar agora numa tentativa de elaborar novas classificações no conjunto das frações que apresentam como resultado números decimais.

Do ponto de vista da aprendizagem, saímos de um lócus e passamos para outro. Com isso, agora temos dois universos para transitar e para estabelecer comparações e conexões: um deles define partição no conjunto do universo $U$, e o outro aponta que é possível definir novas partições a partir da observação dos resultados em um dos subconjuntos definidos pelos alunos.

Matematicamente, essas visões estão relacionadas. Mas, para os alunos, na fase de aprendizagem em que se encontram, tais abordagens são distintas e precisam ser elaboradas e reelaboradas internamente para que possam fazer parte de seu conhecimento. Além disso, somente quando os alunos percebem que essas representações estão relacionadas entre si, de tal forma que tanto o número decimal, com suas características próprias, quanto as frações ordinárias revelam o mesmo conceito, é que conseguem elaborar uma categorização mais ampla. Um exemplo é a categorização feita pelos estudantes e reproduzida a seguir, que classifica os elementos do conjunto formado pelos decimais exatos obtidos a partir da divisão de $n / 2^{b}$ definidos a partir do conceito de múltiplo. Ou seja, classificar as frações considerando ${ }^{3} n \div d$ sendo $d$ um elemento do conjunto $B=\left\{d \in \mathbb{N} / d=2^{b}\right\}$.

\footnotetext{
${ }^{3}$ Inicialmente $n$ significava um número natural (fala e registro da professora). Ao longo da atividade os alunos reinterpretaram seu significando modificando-o para representar a inicial da palavra numerador e $d$, à inicial do denominador.
} 
Para o caso em que o numerador é múltiplo do denominador, ${ }^{4} n=M(d)$, o resultado é sempre um número inteiro. Por outro lado, se o numerador não for múltiplo do denominador, temos muitas possibilidades.

O esquema abaixo sintetiza a visão dos alunos sobre o conjunto dos números decimais obtidos a partir da análise das frações cujo denominador não é múltiplo do denominador. Esse esquema só foi possível de ser organizado dessa forma a partir da discussão com a turma toda.

$$
\frac{n}{2^{b}} \begin{cases}\frac{n}{2} \quad & \{0,5 ; 1,5 ; 2,5 ; 3,5 ; \ldots ; \boldsymbol{a}, \mathbf{5}\} \\
\frac{n}{4} \quad\left\{\begin{array}{l}
\{0,25 ; 1,25 ; 2,25 ; \ldots ; \boldsymbol{a}, \mathbf{2 5}\} \\
\{0,5 ; 1,5 ; 2,5 ; 3,5 ; \ldots ; \boldsymbol{a}, \mathbf{5}\} \\
\{0,75 ; 1,75 ; 2,75 ; 3,75 ; \ldots ; \boldsymbol{a}, \mathbf{7 5}\}
\end{array}\right. \\
\frac{n}{8}\left\{\begin{array}{l}
\{0,125 ; 1,125 ; 1,125 ; 2,125 ; \ldots ; \boldsymbol{a}, \mathbf{1 2 5}\} \\
\{0,25 ; 1,25 ; 2,25 ; 3,25 ; 4,25 ; \ldots ; \boldsymbol{a}, \mathbf{2 5}\} \\
\{0,375 ; 1,75 ; 2,75 ; 3,75 ; \ldots ; \boldsymbol{a}, \mathbf{7 5}\} \\
\{0,5 ; 1,5 ; 2,5 ; 3,5 ; 4,5 ; 5,5 ; \ldots ; \boldsymbol{a}, \mathbf{5}\} \\
\{0,625 ; 1,625 ; 2,625 ; 3,625 ; \ldots ; \boldsymbol{a}, \mathbf{6 2 5}\} \\
\{0,75 ; 1,75 ; 2,75 ; 3,75 ; \ldots ; \boldsymbol{a}, \mathbf{7 5}\} \\
\{0,875 ; 1,875 ; 2,875 ; \ldots ; \boldsymbol{a}, \mathbf{8 7 5}\}
\end{array}\right.\end{cases}
$$

Esquema 1: Síntese dos números decimais com $n \neq M(d)$

O mesmo esquema-resumo foi elaborado para as frações com denominadores cinco, vinte e cinco etc. As observações sobre essas frações e suas representações decimais não diferiram muito daquela elaborada para os denominadores $2^{b}$. Em outras palavras, a representação genérica considerou dois subconjuntos disjuntos partição do conjunto universo entre seus elementos, de forma que: um subconjunto fosse formado pelo resultado inteiro associado ao numerador múltiplo do denominador; e o outro considerasse o número decimal como aquele obtido para o caso em que o numerador não é múltiplo do denominador da fração.

Na comparação das frações de $n / 2^{b}$ com as frações de $n / 5^{b}$, os alunos identificaram que a representação decimal da fração $n / c^{b}$ com o m.d.c. $(n, c)=1$ e $c \in\{2,5\}$, possui $b$ casas decimais. Por que isso acontece todo o tempo? É possível sempre se verificar esse fato? A demonstração nos mostra que sim, como veremos a seguir:

Teorema 1. Sejam n, b, números naturais, e $c \in\{2,5\}$, o m.d.c. $(n, c)=1$. Então, a representação decimal da fração $n / c^{b}$ tem $b$ algarismos decimais (significativos).

Demonstração. Suponhamos que os denominadores sejam potências de cinco. Logo, para $c=5$, temos:

$$
n / 5^{b}=n .2^{b} / 5^{b} 2^{b}=n .2^{b} / 10^{b} .
$$

Como $n$ e 5 são primos entre si, 10 não é divisor de $n .2^{b}$.

Portanto, $n .2^{b} / 10^{b}$ tem exatamente $b$ casas decimais. Estamos considerando no resultado os $b$ algarismos significativos.

Observação 1. Para o caso em que $c=2$, o resultado é obtido de forma análoga.

\footnotetext{
${ }^{4} M(d)$ está sendo entendido como múltiplo de um número natural "d" qualquer. Em particular, $d \in B$.
} 
Os alunos também observaram outra regularidade sobre as frações e a sua representação decimal. Ao compararem frações com o numerador igual a um e os denominadores iguais a potências de base 2 ou 5 com expoentes iguais, a parte decimal de uma das frações é igual ao denominador da outra fração. Por exemplo, $1 / 2$ é igual a 0,5 e $1 / 5$ é igual a 0,2 . Ou seja, a parte decimal de $1 / 2$ é igual ao denominador da fração $1 / 5$, e a parte decimal de $1 / 5$ é igual ao denominador da fração $1 / 2$.

Isto se verifica pelo seguinte fato:

$$
1=10^{b} / 2^{b} .5^{b} \rightarrow 1 / 2^{b}=5^{b} / 10^{b}=0,0 \ldots 05^{b} .
$$

O número de zeros da representação decimal é igual ao expoente $b$ ( $b$ é um número natural) menos a quantidade de algarismos significativos de $5^{b}$, já que $5^{b} / 10^{b}$ tem $b$ casas decimais na sua representação decimal.

O relato dos estudantes foi sistematizado, no quadro negro, numa tabela com as contribuições de todos os alunos da turma da seguinte forma:

\begin{tabular}{|c|c|c|c|c|}
\hline Denominadores (d) & $\mathbf{d = 2}$ & $\mathbf{d}=\mathbf{4}$ & $\mathbf{d = 8}$ & $\mathbf{d = 1 6}$ \\
\hline Frações & $n / 2$ & $n / 4$ & $n / 8$ & $n / 16$ \\
\hline $\mathbf{n = M ( d )}$ & $\mathrm{I}$ & $\mathrm{I}$ & $\mathrm{I}$ & $\mathrm{I}$ \\
\hline & $\mathrm{a}, 5$ & $\mathrm{a}, 25$ & $\mathrm{a}, 125$ & $\mathrm{a}, 0625$ \\
& & $\mathrm{a}, 5$ & $\mathrm{a}, 25$ & $\mathrm{a}, 125$ \\
& & $\mathrm{a}, 75$ & $\mathrm{a}, 375$ & $\mathrm{a}, 1875$ \\
& & $\mathrm{a}, 5$ & $\mathrm{a}, 25$ \\
& & $\mathrm{a}, 625$ & $\mathrm{a}, 3125$ \\
& & $\mathrm{a}, 75$ & $\mathrm{a}, 375$ \\
& & & $\mathrm{a}, 875$ & $\mathrm{a}, 4375$ \\
& & & & e assim por diante. \\
\hline
\end{tabular}

Tabela 1: Regularidades dos decimais com denominadores potência de dois

Na tabela, a letra $\boldsymbol{a}$ antes da vírgula representa, aqui, um número natural qualquer. A letra $\boldsymbol{n}$ significa um número inteiro qualquer colocado no numerador da fração, $\boldsymbol{i}$ representa o resultado inteiro obtido na divisão e $\mathrm{M}(\mathrm{d})$, múltiplo do denominador.

Completadas as três primeiras colunas com os resultados obtidos anteriormente, os alunos começaram a fazer a última coluna calculando apenas as frações com numeradores menores do que o denominador. A justificativa foi:

D: "Ué, a gente já não viu que vai dar sempre alguma coisa ponto outra coisa?"(resposta de um aluno).

Vale a pena reparar que essa frase denota a percepção global do conjunto das frações $n / 16$ e que sua representação decimal terá como parte decimal alguns daqueles números encontrados a partir das frações $n / 16, n \geq 17$. Outra justificativa é a de que "o denominador me dá o $n^{\circ}$ de respostas diferentes", por exemplo: "quando o denominador é dois, tem duas respostas; quando é quatro, tem quatro; quando é oito, tem oito; e assim vai."

B: "No caso do $16 \ldots$..." (interrompido)

A: "Terá 16 respostas diferentes".

$A_{2}$ : "Um inteiro e 15 decimais exatos" (grifo meu para mostrar a alegria do aluno em expressar algo novo e que agora poderá ser usado como objeto da fala e, portanto, de seu conhecimento).

Uma justificativa formal para este fato será feita a seguir: 
Teorema 2. Seja $n$ um número natural, então existem inteiros não negativos q e $r$ tais que $n / 16$ tem representação decimal igual à representação decimal de $q+r / 16$, onde $16>r \geq 0$. E, portanto, o resultado ou é um número inteiro ou é um número decimal em que sua parte decimal é igual a um dos elementos do conjunto $\{1 / 16, \ldots, 15 / 16\}$.

Demonstração. Pelo algoritmo da divisão existem e são únicos $q$ e $r$ tais que:

$$
n=16 . q+r \text {, onde } 16>r \geq 0 .
$$

Assim, $n / 16=q+r / 16$, onde $16>r \geq 0$.

Com este teorema fica claro que toda fração da forma $n / 16$ pode ser representada como a soma de um natural com a fração $r / 16$ onde $16>r \geq 0$. Portanto, para obter sua representação decimal basta calcular as representações decimais das frações $1 / 16, \ldots, 15 / 16$.

Observação 2. A demonstração é feita de forma análoga para outros denominadores. Consideramos o denominador 16 a título de ilustração e para evidenciar a descoberta dos estudantes.

\section{Considerações Finais}

Seria compensador apresentar um mesmo conteúdo usando diferentes formas. No nosso caso, relacionar a representação decimal com a fração através do algoritmo da divisão e usando a calculadora. No primeiro caso, é possível identificar que a operação termina quando o resto é zero e que não é visível no segundo caso. Assim, o uso da calculadora evidenciou alguns aspectos dos racionais que, do outro modo, não ocorreriam.

Neste trabalho, a passagem da representação de fração ordinária para a decimal mostrou algumas propriedades bem particulares, cuja análise só foi possível devido ao grande número de frações representadas em um curto tempo. Por exemplo, os alunos perceberam que o número de casas decimais dependia do valor do expoente do denominador, mas não perceberam que a parte decimal terminava porque o resto da divisão era zero. Fato esse, somente observável quando efetuamos a conta manualmente.

Também observaram questões estudadas anteriormente. Ou seja, identificaram que quanto maior o numerador, menor o resultado obtido; se o numerador for menor do que o denominador, temos um decimal cuja parte inteira é zero; e, nos demais casos, a parte inteira é um número natural diferente de zero.

Os diferentes momentos, trabalho em pequenos grupos, sistematização dos resultados, apresentação para a turma toda e reflexão sobre a produção de todos os grupos, propiciaram aos alunos a oportunidade de esclarecer e aprofundar problemas por eles levantados. Além disso, tanto a participação em pequenos grupos quanto a capacidade individual em cada momento foram outro fator que contribuiu para que os alunos chegassem a determinadas conclusões.

Embora a atividade tenha sido apresentada no contexto da matemática pura, a estratégia usada - investigação em grupo usando calculadora - permitiu desenvolver uma nova dinâmica de trabalho, pois os alunos passaram por vários momentos durante sua execução:

a) a leitura da tarefa e seu reconhecimento como forma de tomar para si a questão colocada pelo professor;

b) o reconhecimento dos procedimentos necessários para a realização da tarefa, relacionando-a com o algoritmo da divisão;

c) o registro de muitas frações e de sua representação decimal, o que favoreceu a análise para a descoberta de regularidades e propriedades bem como a transferência de conceitos (múltiplo de um número) para um novo contexto (o denominador das frações);

d) a justificativa dada para as respostas com a participação dos demais integrantes do grupo de trabalho, os quais contribuíam com seus pontos de vista; 
e) a apresentação e a defesa do ponto de vista de cada aluno no grande grupo;

f) a redação de um relatório que listava as etapas de procedimento do processo investigativo.

A descrição linear das etapas, como feita acima, não ocorre dessa forma em sala de aula, tampouco de forma homogênea em todos os grupos. Esse tipo de atividade exige do professor: disponibilidade para ouvir as diferentes observações feitas por parte dos alunos; atenção para não atropelar as descobertas; adaptação pedagógica; e imposição de novas exigências a seus conhecimentos matemáticos. Com relação ao uso da calculadora, ,

(...) a utilização da calculadora de forma reflexiva e bem planejada pode contribuir para o aprendizado de diversos conteúdos matemáticos, de-senvolvendo a capacidade de investigar ideias matemáticas, resolver problemas, formular e testar hipóteses, induzir, deduzir e generalizar, de modo que os alunos busquem coerência em seus cálculos, comuniquem e argumentem suas ideias com clareza. [2]

Como vimos na análise do trabalho desenvolvido em sala de aula, o uso da calculadora serviu para agilizar os cálculos, apresentar grande variedade de elementos a serem pesquisados, confrontar respostas e confirmar ou refutar conjecturas com maior rapidez.

\section{Referências}

[1] Brekke, G. A decimal number is a pour of whole numbers. In Gutierres, L.P.A. (Ed.). PME 20, Valencia (Espanha),v.2, p.137-144, 1996.

[2] Guinter, A. O uso das Calculadoras nas Aulas de Matemática: concepções de professores, alunos e mães de alunos. 2008. Disponível em:

http://www2.rc.unesp.br/eventos/matematica/ebrapem2008/upload/23-1-A-gt6_ariovaldo_ta.pdf

Acesso em: 10 de maio de 2011.

[3] Kindel, D. S. A multiplicação: uma reflexão sobre o uso de calculadoras na quinta série. Boletim Gepem, n. 45, p. 54-62, 2004.

[4] Kindel, D. S. Discutindo os racionais na $7^{a}$ série visando a noção de densidade. 196fls. Dissertação (Mestrado em Educação Matemática), Universidade Santa Úrsula, Rio de Janeiro, 1998.

[5] Mason, J. O quê, o porquê e o como em matemática. In: Abrantes, I. N. P.; Leal, I. C.; Ponte, J. P. (eds.). Investigar para aprender matemática.APM (Lisboa), p. 15-24, 1996.

[6] Ponte, J. P.; Brocardo, J.; Oliveira, H.. Investigações Matemáticas em sala de aula. Belo Horizonte: Autêntica, 2003.

Dora Kindel

UFRRJ

<soraiakindel@yahoo.com.br>

Erika Favoretto

Faculdade Alvorada de Educação Física e Desporto

$<$ erikafavoretto@hotmail.com>

Recebido: 2013

Publicado: 2013 